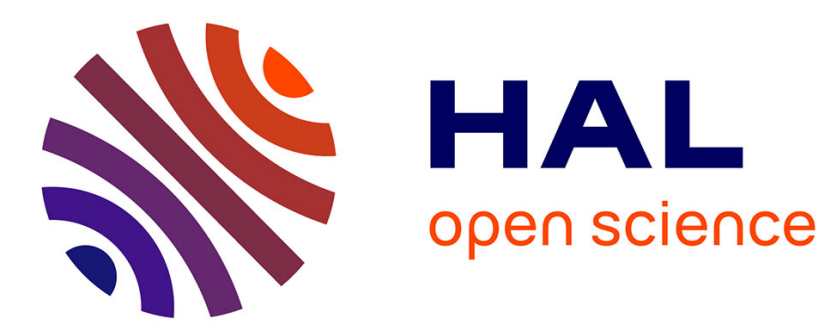

\title{
Déterminisme génétique du débit de lait au cours de la traite des chèvres
}

\author{
G. Ricordeau, J. Bouillon, P. Leroy, J.M. Elsen
}

\section{To cite this version:}

G. Ricordeau, J. Bouillon, P. Leroy, J.M. Elsen. Déterminisme génétique du débit de lait au cours de la traite des chèvres. Productions Animales, 1990, 3 (2), pp.121-126. hal-00895894

\section{HAL Id: hal-00895894 \\ https://hal.science/hal-00895894}

Submitted on 1 Jan 1990

HAL is a multi-disciplinary open access archive for the deposit and dissemination of scientific research documents, whether they are published or not. The documents may come from teaching and research institutions in France or abroad, or from public or private research centers.
L'archive ouverte pluridisciplinaire HAL, est destinée au dépôt et à la diffusion de documents scientifiques de niveau recherche, publiés ou non, émanant des établissements d'enseignement et de recherche français ou étrangers, des laboratoires publics ou privés. 
INRA Prod. Anim., 1990, 3 (2), $121-126$
G. RICORDEAU, J. BOUILLON*, P. LEROY ${ }^{*}$, J.M. ELSEN

Avec la collaboration technique de A. LAJOUS

INRA Station d'Amélioration Génétique des Animaux

B.P. 27, 31326 Castanet Tolosan Cedex

* Station de Testage Caprin

Moissac

48110 Sainte-Croix-Vallée-Française

* INRA Station de Génétique

Quantitative et Appliquée

78352 Jouy-en-Josas Cedex
Déterminisme génétique du débit de lait au cours de la traite des chèvres

Le débit de lait au cours de la traite des chèvres n'est pas un objectif prioritaire de sélection, mais en influençant le temps de traite, il peut présenter un réel intérêt économique, notamment dans les grands troupeaux qui deviennent de plus en plus nombreux. Prendre en compte cet objectif dans le schéma national de sélection est non seulement souhaitable, mais désormais possible, dans la mesure où le caractère « haut débit » semble conditionné par un gène majeur.

Chez les femelles laitières, on observe une grande hétérogénéité du débit de lait au cours de la traite, caractère dont le déterminisme n'est peut être pas uniquement de type polygénique. Ainsi, chez les bovins, les différents critères retenus (débit des 2 premières minutes, débit moyen ou maximum) ont en général une héritabilité moyenne $(0,25$ à 0,50 : Venge 1963 , Williams et al 1984, Schneeberger et Hagger 1985, Petersen et al 1986), mais quelques études indiquent des héritabilités de 0,60 à 0,80 (Wilson 1963, Politiek 1969). D'autre part, Phillips (1987) signale que la quantité de sti-

\section{Résumé}

Le débit de lait au cours de la $1^{\mathrm{e}}$ minute de traite des chèvres varie de 0,3 à $2,4 \mathrm{~kg}$. L'analyse des descendances des boucs à la Station de Moissac sur 8 campagnes ( 1585 lactations de 893 chèvres nées de 97 pères et 487 mères) a fait apparaître 3 catégories de pères, ce qui nous a conduit à envisager l'hypothèse d'un locus à effet majeur, avec 2 allèles en ségrégation, un allèle +, normal et partiellement dominant, et un allèle hd, récessif, responsable d'un haut débit de traite.

Les différents tests utilisés montrent que cette hypothèse est vraisemblable et que le déterminisme génétique du débit est de type mixte. Le débit moyen des chèvres homozygotes hd/hd est supérieur de $84 \%$ à celui des chèvres homozygotes $+1+$ (respectivement 1,48 vs $0,80 \mathrm{~kg} / \mathrm{min}$ ), celui des hétérozygotes hd/+ étant de 0,94 $\mathrm{kg} / \mathrm{min}$, ce qui correspond à une dominance de 0,61 . La fréquence de l'allèle hd serait de 0,39 dans l'échantillon étudié, l'héritabilité résiduelle du débit étant de $\mathbf{0 , 4 8}$.

La sélection des chèvres à haut débit est donc possible et souhaitable. Elle peut être réalisée, dans un premier temps, en limitant les mesures de débit aux " mères à boucs " (chèvres les mieux indexées), afin de détecter celles qui ont un débit supérieur à $1,46 \mathrm{~kg} / \mathrm{min}$ (supposées toutes homozygotes hd/hd) et de produire rapidement des jeunes mâles susceptibles d'être homozygotes hd/hd. muli nécessaire à l'éjection du lait pourrait avoir un déterminisme simple. Chez les brebis laitières, la cinétique de la descente du lait a mis en évidence 2 à 4 types de débits, avec des différences entre races et entre pères (Labussière et Ricordeau 1970, Hatziminaoglou et Sinapis 1987, Purroy et al 1987, Fernandez et al 1989, Mayer et al 1989).

Chez les caprins, la traite a fait l'objet de quelques études relatives aux paramètres de la machine à traire, à l'organisation du travail de traite, aux mesures du canal du trayon ou des débits de traite, à l'éjection du lait (Ricordeau et Labussière 1970, Mosdol 1980, Pettersen 1981, Le Du et Benmederbel 1984, Le Du 1989, Lyons 1989), mais les études génétiques sur la facilité de traite sont rares.

Depuis 1968, les chèvres nées des boucs en testage à la Station de Moissac (Lozère) font l'objet de mesures systématiques. Les premiers résultats montrent que le débit est un caractère très héritable (Bouillon et Ricordeau 1981), cependant la sélection pratiquée pour améliorer ce débit n'a pas donné les résultats espérés. On constate en effet que les mères à débit élevé (plus de 1,2 kg de lait pendant la 1ère minute) donnent à la fois des filles à haut et bas débit. Par ailleurs, l'analyse des descendances fait apparaître 3 catégories de pères: les premiers ont des filles dont le débit est pratiquement toujours inférieur à $1,2 \mathrm{~kg} / \mathrm{min}$, quel que soit le débit de leurs conjointes; les seconds ont des filles à débit élevé lorsqu'ils sont accouplés à des conjointes à haut débit ; les troisièmes ont des filles à haut et à bas débit avec des mères à haut débit. Ces observations nous ont conduit, 
dès 1985, à envisager l'hypothèse de l'existence d'un locus à effet majeur sur le débit de traite des chèvres, avec 2 allèles en ségrégation : un allèle + , normal et dominant, et un allèle hd, récessif et responsable d'un haut débit de traite.

Cet article résume les résultats obtenus dans la vérification de cette hypothèse. Les analyses détaillées sont présentées par ailleurs (Le Roy 1989, Ricordeau et al 1989).

\section{1 / Conditions expérimentales}

Le troupeau de la station est fermé pour la voie femelle et ouvert pour la voie mâle (insémination artificielle). Les pères sont des boucs en testage, de type Alpin ou Saanen, sélectionnés sur les index quantité et taux de protéines de leurs parents, et contrôlés sur leur aptitude à la reproduction dans 2 centres de production de semence. Comme la classification des femelles repose essentiellement sur la couleur de la robe, nous avons regroupé l'ensemble des données, en prenant toutefois en compte le poids à la mise bas pour éliminer les différences de format, les descendances nées de pères Saanen étant en moyenne plus lourdes que celles issues de pères Alpins. La première mise bas a lieu à 13 mois, la deuxième et les suivantes à 2 ans, 3 ans, etc... de sorte que l'âge en années équivaut au numéro de lactation.

L'échantillon disponible représente 893 chèvres, contrôlées de 1981 à 1988 et ayant réalisé un total de 1585 lactations ( 1 à 6 par chèvre). Elles sont issues de 97 pères et 487 mères, avec au moins 5 filles par père.

Le débit correspond à la quantité de lait obtenue au cours de la première minute de traite, à partir de la pose des gobelets (en $\mathrm{kg} / \mathrm{min}$ ). Les contrôles sont effectués à la même période sur l'ensemble du troupeau (en avril), c'est-à-dire en moyenne à 60 jours de lactation pour les primipares, à 90 jours de lactation pour les adultes. Ils sont réalisés à la traite du matin, sur 2 ou 3 jours consécutifs pour chaque animal. Les paramètres de la machine sont fixés à un rapport de pulsation de $2 / 1$, une vitesse de pul- sation de $90 / \mathrm{min}$ et un vide de 33 ou $35 \mathrm{~cm}$ de $\mathrm{Hg}$. Par ailleurs, nous disposons des performances obtenues pour les 100 premiers jours de lactation grâce au contrôle laitier réalisé tous les 14 jours: quantité de lait en $\mathrm{kg}$ (QL 100), taux butyreux (TB 100) et taux de protéines (TP 100) en $\mathrm{g} / \mathrm{kg}$.

\section{2 / Effets fixés et paramètres génétiques}

Ils ont été estimés par analyse de variance, dans l'hypothèse d'absence de gène majeur, sur la variable débit, mais aussi sur les autres variables de production laitière prises en quelque sorte comme variables témoins (tableau 1). L'effet « campagne » est non significatif pour le débit et très significatif pour les 3 autres variables, mais seul le TP 100 augmente régulièrement de 1981 à 1988. L'effet " âge », ou numéro de lactation, est significatif sur le débit, mais seulement de 2 à 3 ans et plus, et très significatif sur la QL $100(+55 \mathrm{~kg}$ entre 1 et 2 ans et + $40 \mathrm{~kg}$ de 2 à 3 ans et plus), le TB 100 et le TP 100 de 1 à 2 ans $(+2,6$ et $+0,5 \mathrm{~g} / \mathrm{kg})$. L'effet " poids à la mise bas " n'est significatif que sur la quantité de lait (coefficient de régression linéaire de $1,2 \mathrm{~kg}$ de lait en $100 \mathrm{j}$ par $\mathrm{kg}$ de poids vif).

Les paramètres génétiques des variables de production laitière à 100 jours (tableau 2) sont légèrement supérieurs à ceux observés en fermes (Boichard et al 1989). Cependant, les tendances classiques sont respectées avec une héritabilité moyenne de la quantité de lait et forte des 2 taux, une opposition génétique entre quantité et taux, surtout marquée pour le taux protéique $(-0,45)$ et une corrélation génétique positive de 0,57 entre les 2 taux.

En ce qui concerne le débit de traite, le fait marquant est l'obtention d'une héritabilité de $0,97( \pm 0,16)$. Le débit apparaît par ailleurs en corrélation génétique positive avec la quantité de lait $(+0,31)$ et négative avec le taux protéique $(-0,24)$, alors que la liaison avec le taux butyreux est nulle tant du point de vue génétique que phénotypique.

Tableau 1. Moyennes des moindres carrés ( \pm erreur-standard) pour les effets fixés et coefficient de régression sur la variable "poids à la mise bas" (b).

\begin{tabular}{|c|c|c|c|c|c|}
\hline \multicolumn{2}{|l|}{ EFFET } & $\begin{array}{c}\text { DEBIT } \\
(\mathrm{kg} / \mathrm{min})\end{array}$ & $\begin{array}{c}\text { QL100 } \\
\text { (kg) }\end{array}$ & $\begin{array}{l}\text { TB } 100 \\
(\mathrm{~g} / \mathrm{kg})\end{array}$ & $\begin{array}{l}\text { TP100 } \\
\text { (g/kg) }\end{array}$ \\
\hline Campagne & $\begin{array}{l}1981 \\
1982 \\
1983 \\
1984 \\
1985 \\
1986 \\
1987 \\
1988\end{array}$ & $\begin{array}{c}1,02 \pm 0,04 \\
1,04 \pm 0,03 \\
1,00 \pm 0,03 \\
1,03 \pm 0,02 \\
1,01 \pm 0,02 \\
1,02 \pm 0,02 \\
0,98 \pm 0,02 \\
1,09 \pm 0,02 \\
\text { (ns) }\end{array}$ & $\begin{array}{c}335 \pm 6 \\
378 \pm 5 \\
354 \pm 4 \\
331 \pm 4 \\
334 \pm 4 \\
329 \pm 4 \\
320 \pm 4 \\
328 \pm 4 \\
\left({ }^{* \star}\right)\end{array}$ & $\begin{array}{c}35,0 \pm 0,4 \\
35,5 \pm 0,4 \\
35,6 \pm 0,3 \\
35,5 \pm 0,3 \\
37,5 \pm 0,3 \\
36,7 \pm 0,3 \\
34,7 \pm 0,3 \\
33,5 \pm 0,3 \\
(* * *)\end{array}$ & $\begin{array}{l}27,1 \pm 0,2 \\
27,3 \pm 0,2 \\
28,0 \pm 0,1 \\
27,1 \pm 0,1 \\
27,7 \pm 0,1 \\
27,5 \pm 0,1 \\
28,9 \pm 0,1 \\
29,5 \pm 0,1 \\
\left({ }^{* \star}\right)\end{array}$ \\
\hline $\begin{array}{c}\mathrm{N}^{\circ} \mathrm{de} \\
\text { lactation }\end{array}$ & $\left\{\begin{array}{c}1 \\
2 \\
\geq 3\end{array}\right.$ & $\begin{array}{c}1,02 \pm 0,02 \\
1,06 \pm 0,02 \\
0,97 \pm 0,02 \\
\left({ }^{* *}\right)\end{array}$ & $\begin{array}{c}290 \pm 2 \\
345 \pm 3 \\
385 \pm 4 \\
(\star \star \star)\end{array}$ & $\begin{array}{c}33,7 \pm 0,2 \\
36,3 \pm 0,2 \\
36,5 \pm 0,3 \\
\text { (**) }^{* * *}\end{array}$ & $\begin{array}{c}27,6 \pm 0,1 \\
28,1 \pm 0,1 \\
28,1 \pm 0,1 \\
(* * *)\end{array}$ \\
\hline (b) & & (ns) & $1,2(* * *)$ & (ns) & (ns) \\
\hline
\end{tabular}


Tableau 2. Héritabilités (sur la diagonale), corrélations phénotypiques (au-dessus) et génétiques (audessous) ( \pm erreur-standard).

\begin{tabular}{|lrrrr|}
\hline Caractères & DEBIT & QL 100 & TB 100 & TP 100 \\
\hline DEBIT & $0,97 \pm 0,16$ & $0,17 \pm 0,03$ & $-\mathbf{0 , 0 6} \pm 0,04$ & $-0,11 \pm 0,04$ \\
QL 100 & $0,31 \pm 0,17$ & $0,31 \pm 0,09$ & $-0,23 \pm 0,14$ & $-0,46 \pm 0,02$ \\
TB 100 & $-0,09 \pm 0,15$ & $-0,28 \pm 0,18$ & $0,74 \pm 0,14$ & $0,33 \pm 0,03$ \\
TP 100 & $-0,24 \pm 0,14$ & $-0,45 \pm 0,15$ & $\mathbf{0 , 5 7} \pm 0,11$ & $0,69 \pm 0,13$ \\
\hline
\end{tabular}

\section{3 / Vérification de l'hypothèse gène majeur}

La distribution du débit (figure 1a) est non normale, dissymétrique, leptokurtique (c'est-àdire pointue) et l'hypothèse d'un mélange de 2 distributions normales ne peut être rejetée. Les variances intra famille de père sont hétérogènes et le test de Fain (1978), qui mesure la curvilinéarité de la liaison entre moyennes et variances intra famille, est hautement significatif. L'analyse de ségrégation (Elston et Stewart 1971) qui teste différentes hypothèses de transmission génétique en comparant la vraisemblance des distributions familiales observées, permet de conserver l'hypothèse d'une hérédité polygénique pour les 3 variables QL 100, TB 100 et TP 100, mais confirme celle d'un déterminisme mixte pour le débit (tableau 3). Le gène hd pour l'allèle haut débit serait pratiquement récessif. Les chèvres homozygotes hd/hd ont un débit moyen de $1,48 \mathrm{~kg}$ par min, contre 0,80 pour les chèvres homozygotes $+/+$, soit une différence de 2,5 écarts-types phénotypiques $(\sigma=0,26 \mathrm{~kg} / \mathrm{min}$. $)$. Les chèvres hétérozygotes $\mathrm{hd} /+$ ont un débit légèrement supérieur aux homozygotes à faible débit $(0,94$ vs 0,80$)$, ce qui correspond à une dominance de 0,61 . La fréquence de l'allèle favorable (hd) est de l'ordre de 0,39 dans la population des reproducteurs. Enfin, sous cette hypothèse de déterminisme mixte, le débit a une répétabilité de 0,73 et une héritabilité résiduelle de 0,48 (tableau 3).

\section{4 / Perspectives}

Les débits moyens estimés dans cette étude pour les chèvres $+/+$ et hd $/+(0,80$ et $0,94 \mathrm{~kg} /$ min.) sont peu différents de ceux observés sur chèvres Saanen et Alpine dans d'autres situations : 0,94 pour Le Du et Benmederbel (1984); 0,69 à 0,79 pour Mikus et Mikus (1988); 0,67 pour Cicogna (1984). Un contrôle effectué par Bridoux et Bouillon (1986) sur 832 chèvres Alpine et Saanen dans 52 élevages des DeuxSèvres, permet d'avoir une idée assez précise des débits observés en fermes : $0,97 \mathrm{~kg} / \mathrm{min}$. en 1ère lactation, 0,87 pour les lactations 2 à 4 , et 0,76 pour les lactations 5 et plus, avec $7 \%$ de chèvres de tous âges ayant un débit supérieur à $1,4 \mathrm{~kg} / \mathrm{min}$. (figure $1 \mathrm{~b}$ ).

Compte tenu de la différence observée entre les deux types d'homozygotes, il importe maintenant de multiplier les vérifications expérimentales, aussi bien en station qu'en fermes, et d'étudier de façon plus analytique les caractéristiques de traite, pour expliquer les mécanismes d'action du gène hd et mesurer les éventuels effets sur la qualité de la traite (égouttage, sensibilité aux mammites,...).

Même si le débit n'est pas un objectif prioritaire de sélection, son incidence sur le temps

Figure 1. Distribution du débit de lait au cours de la première minute de traite des chèvres.

(a) Chèvres Alpine et Saanen à la Station de Moissac.

(b) 832 chèvres Alpines et Saanen dans 52 élevages : $0,97 \mathrm{~kg} / \mathrm{min}$ en lactation $1(n=285) ; 0,85$ en lactation 2 à $4(n=314) ; 0,76$ en lactation 5 et plus $(n=233)$ : d'après Bridoux et Bouillon 1986.

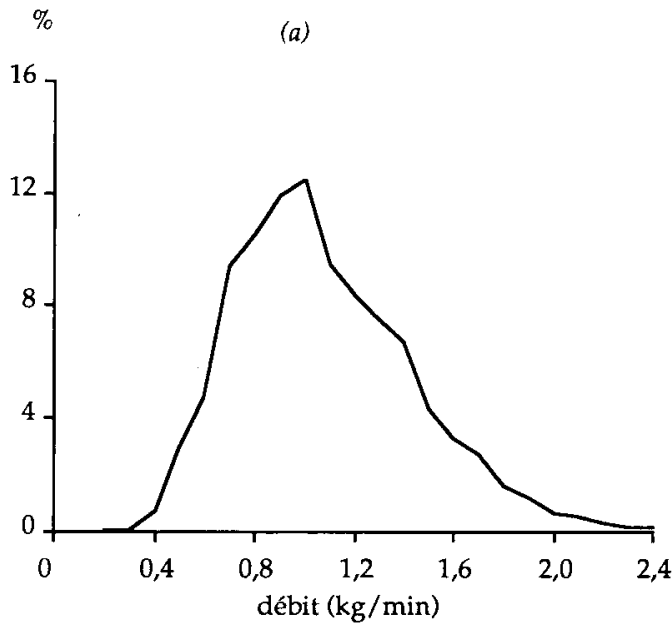

$\% \quad(b)$

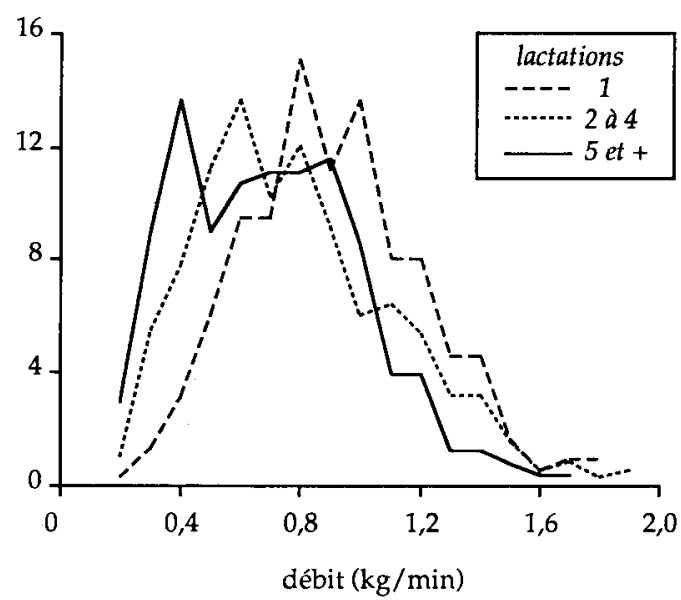

Les différents tests montrent que l'hypothèse d'un locus à effet majeur est vraisemblable. $A$ ce locus, 2 allèles en ségrégation : un « débit normal » dominant et un " haut débit » récessif. 


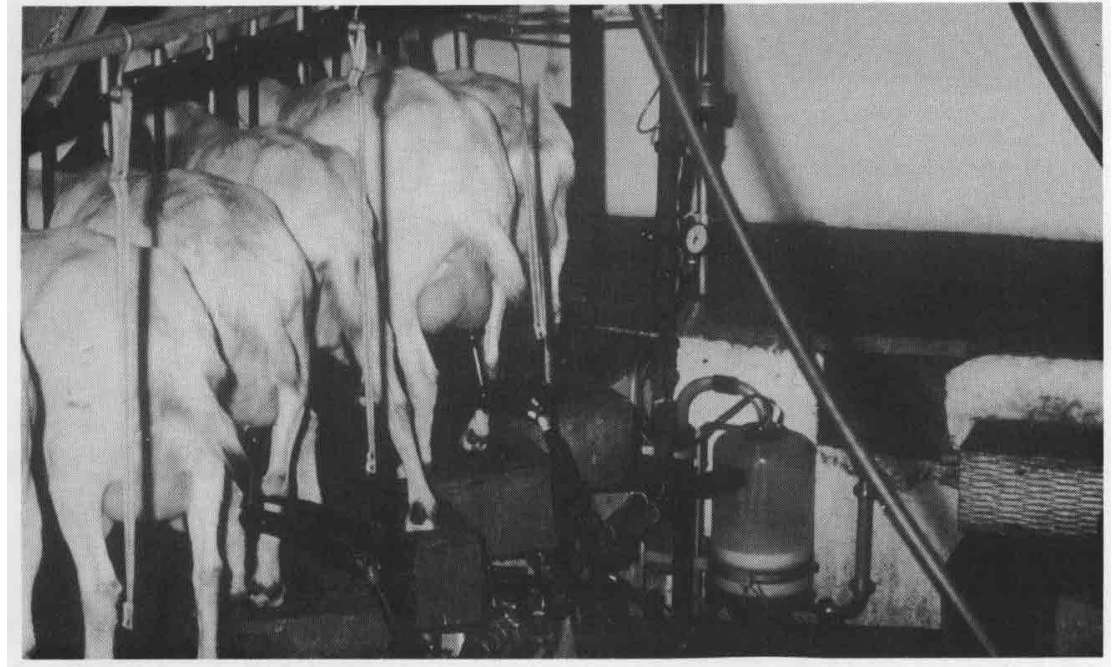

Les chèvres dont le débit est supérieur à $1,46 \mathrm{~kg} / \mathrm{min}$ peuvent être considérées comme homozygotes " haut débit ». de traite peut présenter un intérêt économique non négligeable, notamment dans les grands troupeaux où le passage simultané des chèvres rapides et lentes dans les salles de traite (quai droit, en épi ou avec rotolactor) perturbe le travail des trayeurs. En effet, depuis une vingtaine d'années, l'élevage caprin a considérablement évolué. L'effectif moyen de chèvres en lactation par élevage est passé de 31 en 1968 (15 000 chèvres contrôlées) à 50 en 1978 (73 000), pour atteindre 76 en 1988 (216 000) et même 95 dans les 3 départements des Deux-Sèvres, de la Vienne et de Vendée qui regroupent la moitié des effectifs contrôlés (Sigwald et al 1989). Prendre en compte le débit dans le programme de sélection est donc un objectif souhaitable, connaissant le déterminisme du caractère et la marge de progrès possible.

\section{5 / Possibilités de sélection}

Le fait que le caractère haut débit soit récessif a plusieurs conséquences : il permet de bien distinguer les chèvres homozygotes hd/hd, mais ne permet pas de typer aisément les boucs; il oblige à choisir des parents à haut débit si l'on veut produire uniquement des filles et des fils à haut débit.

Tout d'abord, il s'agit de distinguer les chèvres homozygotes à haut débit. Avec les paramètres de fonctionnement de la machine à traire utilisés dans cette étude (notamment un rapport de pulsation de $2 / 1$ ), on peut estimer que ce sont pratiquement toutes celles dont le débit est supérieur à $1,46 \mathrm{~kg} / \mathrm{min}$, si l'on veut éviter la confusion avec les chèvres $+1+$ et surtout hd/+. Cependant, compte tenu des effets parasites (élevage-machine à traire; âge-stade de lactation ; différences entre quartiers dans le délai d'arrivée du lait depuis la pose des gobelets), on peut être moins sévère et retenir un seuil légèrement inférieur en répétant si possible les mesures.

Il faut ensuite déterminer le génotype des boucs d'insémination à partir du débit de leurs filles (Elsen et al 1988). La solution idéale serait de contrôler toutes les chèvres, ce qui sera réalisable dès que le contrôle laitier pourra s'équi-

Tableau 3. Paramètres déduits de l'analyse de ségrégation de la variable DEBIT.

\begin{tabular}{|c|c|c|}
\hline Hypothèse & $\begin{array}{c}\text { Déterminisme } \\
\text { polygénique }\end{array}$ & $\begin{array}{c}\text { Déterminisme } \\
\text { mixte }\end{array}$ \\
\hline$\mu_{0}$ & 0,981 & - \\
\hline$\mu_{1}(+/+)$ & & 0,804 \\
\hline$\mu_{2}(\mathrm{hd} /+)$ & & 0,936 \\
\hline$\mu_{3}(\mathrm{hd} / \mathrm{hd}$ ) & & 1,479 \\
\hline fréquence $(+/+)$ & & 0,406 \\
\hline fréquence (hd/+) ) & & 0,412 \\
\hline$\sigma_{\mathrm{e}}($ résiduel) & 0,137 & 0,138 \\
\hline$\sigma_{\mathrm{c}}$ (chèvre) & 0,176 & 0,132 \\
\hline$\sigma_{g}$ (génétique) & 0,269 & 0,182 \\
\hline $\begin{array}{l}\text { Effet du } n^{\circ} \\
\text { de lactation }\end{array}\left\{\begin{array}{l}1^{r} \\
2^{n}\end{array}\right.$ & $\begin{array}{l}0,113 \\
0,036\end{array}$ & $\begin{array}{l}0,113 \\
0,036\end{array}$ \\
\hline
\end{tabular}

$\mu_{0}=$ débit moyen ajusté dans l'hypothèse " déterminisme polygénique ».

$\mu_{1}, \mu_{2}, \mu_{3}=$ débits moyens ajustés des chèvres $+/+, \mathrm{hd} /+$ et hd/hd dans l'hypothèse « déterminisme mixte ". La dominance est estimée à partir de la moyenne des 3 génotypes par :

$\mathrm{d}=\left(\mu_{2}-\frac{\mu_{1}+\mu_{3}}{2}\right) /\left(\mu_{1}-\frac{\mu_{1}+\mu_{3}}{2}\right)$

Héritabilité, $h^{2}=\sigma_{g}^{2} /\left(\sigma_{g}^{2}+\sigma_{c}^{2}+\sigma_{0}^{2}\right)$

Répétabilité, $\mathrm{r}=\left(\sigma_{\mathrm{g}}^{2}+\sigma_{\mathrm{c}}^{2}\right) /\left(\sigma_{\mathrm{g}}^{2}+\sigma_{\mathrm{g}}^{2}+\sigma_{\mathrm{c}}^{2}+\sigma_{\mathrm{e}}^{2}\right)$

Note sur l'analyso de ségrégation. C’est une technique utilisée depuis une vingtaine d'années, essentiellement en génétique humaine, pour choisir entre des hypothises rälatives al mode de Iransmission des caractères: hypothèse monogénique (caractère contrôlé par un seul gène à effet majeur], polygénique (contrôlé par un grand nombre de genes à petits effets), mixte (monogénique + polygénique), sporadique (pas de transmission génétique). Chacune de cess hypotheses repose sur des paramètres (moyennes, variances, fréquences génotypiques...) qui sont estimés par maximisation de la vraisemblance des observalions. Une hypothèse est retenue si la vraisemblance qui lui correspond est grande par rapport à celle des autres hypothèses. Le crilère statistique pour décider de retenir ou rejeter une hypothèse est le rapport de vraisemblance qui, sous certaines ait une loi statistique bien connue. La décision se prend donc en comparant la valeur de ce rapport à un seuil donné par une table de rétérence. Pour plus de précisions sur cette méthode, consulter Le Roy. Elsen et Knott, 1989. 
per d'éprouvettes automatisées permettant d'obtenir à la fois la quantité de lait, un échantillon de lait et une ou plusieurs mesures du débit (cf modèle déposé INRA, Guillouet et al 1988). Cependant, la détection des boucs homozygotes à haut débit risque d'être imprécise, si ceux-ci sont accouplés essentiellement à des mères à faible débit, homozygotes $+1+$ par exemple. Pour détecter efficacement les boucs hd/hd, il faudrait donc les tester sur des mères homozygotes à haut débit, afin d'obtenir 5 à 10 filles et vérifier qu'elles sont bien toutes hd/hd, à condition bien sûr qu'il n'y ait aucune erreur de filiation.

En attendant, on peut envisager de produire, puis de tester, des jeunes boucs supposés homozygotes hd/hd, en accouplant des fils de mères à haut débit à des chèvres à haut débit. Cette solution permettrait de limiter le contrôle du débit aux seules "mères à boucs" dans les élevages connectés (c'est-à-dire les chèvres les mieux indexées dans les élevages pratiquant régulièrement l'insémination artificielle) pour détecter celles ayant un débit supérieur à 1,46 $\mathrm{kg} / \mathrm{min}$. Comme ces dernières sont relativement peu nombreuses ( $15 \%$ dans notre échantillon ; $7 \%$ dans l'échantillon contrôlé par Bridoux et Bouillon en 1986), il faudrait donc retenir un maximum de leurs fils, sans toutefois diminuer sensiblement la pression de sélection sur les 2 principaux critères de sélection (la quantité de matière protéïque et le taux de protéines), ce qui suppose une augmentation du nombre de mâles mis en testage. En comparant l'index débit de ces boucs à celui de leurs parents, on aurait ainsi la possibilité de vérifier en fermes le déterminisme génétique de ce caractère et d'augmenter le nombre de boucs améliorateurs.

\section{Conclusion}

Les préoccupations essentielles des éleveurs de Caprins portent sur l'amélioration de la quantité de protéines, du taux de protéines et de la facilité de traite. La découverte du polymorphisme des caséines (Grosclaude et al 1987) et la mise en évidence d'un gène majeur sur le débit de traite, sont des acquis qui peuvent être déterminants dans les années à venir.

\section{Remerciements}

M. G. Bridoux, Directeur du Syndicat pour l'amélioration des performances des femelles laitières des Deux Sèvres (SAPERFEL), a accepté, dès 1985, de mettre en place un contrôle du débit dans 52 élevages de son département. Nous le remercions pour l'intérêt qu'il a apporté à la mise en place et à la réalisation de ce travail expérimental.

\section{Références bibliographiques}

BOICHARD D., BOULOC N., RICORDFAU G., PIACERE A., BARILLET F., 1989. Genetic parameters for first lactation dairy traits in the Alpine and Saanen goat breeds. Genet. Sel. Evol., 21, 205-215.

BRIDOUX G., BOUILLON I., 1986. Observations préliminaires sur le débit de traite des chèvres Alpine et Saanen dans 52 élevages du département des Deux Sèvres. Document SAPERFEL, non publié.
BOUILLON J., RICORDEAI G., 1981. Selection des chèvres sur l'aptitude à la traite. In Gèmes Journées de la Recherche Ovine et Caprine, INRA-ITOVIC, Ed. 91-98.

CICOGNA M., 1984. Research on udder shape of Chamois coloured goats and their aptitude for machine milking. Rivista di Zootechnia e Veterinaria, 12, 376-381 (en Italien].

ELSEN I.M., VU TIEN KHANG J., LE ROY P., 1988. A statistical model for genotype determination at a major locus in a progeny test design. Génét. Sél. Evol., 20, 211226.

ELSTON R.C., STEWART O., 1971. A general model for the genetic analysis of pedigree data. Hum. Hered. 21, 523-542.

FAIN P.R., 1978. Characteristics of simple sibship variance tests for the detection of major loci and application to height, weight and spatial performance. Ann. Hum. Genet., 42, 109-120.

FERNANDEZ N., CAJA G. TORRES A., MOLINA M.P., PERIS C., 1989. Kinetic of milk emission in Manchega sheep. I. Parameters of emission curves during mechanical milking. Invest. Agrar. Prod. Sanid. Anim., 4, 9-21 (en Espagnol).

GROSCLAUDE F., MAHE M.F. BRIGNON G., DI STASIO L., JEUNET R., 1987. A Mendelian polymorphism underlying quantitative variations of goat a S1-casein. Génét. Sél. Evol., 19, 399-412

GUILLOUET P., RICARD E., ROUSSELY M., POIVEY J.P., BIBE B BARILLET F 1988. Conception d'un système de contrôle laitier automatisé pour les ovins et caprins laitiers. Dossier INRA-août 1988.

HATZIMINAOGLOU J., SINAPIS F., 1987. The relation of the kinetic of milk flow and the drop of milk production of ewes observed immediately after weaning. Animal Sci. Review, 5, 49-61 (en grec).

LABUSSIERE J., RICORDEAU G., 1970. Aptitude à la traite mécanique des brebis de race Préalpes du Sud et croisées Frisons x Préalpes; étude à différents stades de lactation. Ann. Zootech., 19, 159-190.

LE DU J., 1989. La traite mécanique des chèvres. Prod. Anim., 2(1), 31-38.

LE DU J. BENMEDERBEL B. 1984. Aptitude des chèvres de race Saanen à la traite mécanique. Relations avec les caractéristiques physiques du trayon. Ann. Zootech., 33, 375-384.

LE ROY P., 1989. Méthodes de détection de gènes majeurs. Application aux animaux domestiques. Thèse Université Paris XI. Orsay.

LE ROY P. ELSEN J.M. KNOTT S., 1989. Comparison of four statistical methods for detection of a major gene in a progeny test design. Genet. Sel. Evol., 21, 341-357.

LYONS D.M., 1989. Individual differences in temperament of dairy goats and the inhibition of milk ejection. Appl. Anim. Behav. Sci., 22, 269-282.

MAYER H. WEBER F. SEGESSEMANN $V$., 1989. A method to record and define milk flow curves of sheep during routine machine milking. 4th Intern. Symposium on machine milking of small ruminants, 13-19 Sept.1989, Tel Aviv, Israël, p. 101 (Abstr).

MIKUS M., MIKUS M., 1988. The effect of age on the rate of milk release in the machine milking of goats Zivocisna Vyroba, 61, 661-670 (en Tchèque; résumé en anglais).

MOSDOL G., 1980. Teat canal diameter and milking-out characteristics in the goat. Thesis Veterinary College of Norway, Oslo, 1980, $138 \mathrm{pp}$

PETERSEN M. L., HANSEN L B., YOUNG C.W., MILLER K.P. 1986. Rates of milk flow and milking times resulting from selection for milk yield.J. Dairy Sci., 69, 556-563.

PETTERSEN K.E., 1981. Effect of an udder health programme on goats. Thesis, Norges Veterinaerhogskole, Bodo, Norway (en Norvégien).

PHILLIPS D.S.M., 1987. Studies on pre-milking preparation. 10. Long-term change in yield response to pre-milking preparation. N.Z. J. Agric. Res., 30, 317-323.

POLITIEK R.D., 1969. Selection on ease of milking worth while? World Rev, Anim. Prod., 94-98. 
PURROY A., MARTIN J.L., JURADO J., 1987. The aptitude of Churro ewes for machine milking. II. Kinetics of milk ejection. Invest. Agrar. Prod. Sanid. Anim., 2, 53-63.

RICORDEAU G., LABUSSIERE J., 1970. Traite à la machine des chèvres. Comparaison de 2 rapports de pulsation et efficacité de la préparation de la mamelle avant la traite. Ann. Zootech., 19, 37-43.

RICORDEAU G., BOUILLON J., LE ROY P., ELSEN J.M., 1989. Mise en évidence d'un gène à effet majeur sur le débit de traite des chèvres. 4th Intern. Symposium on machine milking of small ruminants, September 13-19, 1989. Tel-Aviv, Israël.

SCHNEEBERGER M., HAGGER C.H., 1985. Sire evalua- tion for milkability traits in Swiss Braunvieh. Liv. Prod. Sci., 13, 219-227.

SIGWALD J.P. MAINGOT A., LEQUENNE D, 1989. Résultats de la campagne de contrôle laitier 1988. Document ITOVIC FNOCL. 149 Rue de Bercy, 75595 Paris Cédex 12.

VENGE O., 1963. Investigations of milking characteristics in Swedish Red and White Cattle. Lantbrukshögsk. Ann., 29, 187-214.

WILLIAMS C.B., BURNSIDE E.B., SCHAEFFER L.R., 1984. Genetic and environmental parameters of two field measures of milking speed. J.Dairy Sci., 67, 1273-1280.

WILSON G.F., 1963. Some aspects of machine milking rate. J. Dairy Res., 30, 191-196.

\section{Summary}

Genetic of milk flow in Alpine and Saanen goats.

The first minute of milk flow of goats varies between 0.3 and $2.4 \mathrm{~kg}$. Observation of halfsib families in the Moissac progeny-test station during 8 years (1585 lactations of 893 goats born from 97 sires and 487 dams) showed 3 types of sires, suggesting the possible existence of a major gene with 2 segregating alleles, + , normal and partially dominant, and hd, recessive, increasing milk flow.

This hypothesis of a mixed inheritance was confirmed using different test statistics. The average milk flow of homozygous hd/hd goats is $84 \%$ higher than the $+/+$ mean $(1.48$ vs $0.80 \mathrm{~kg} / \mathrm{min}$, respectively), the value of hetero- zygous hd/+ averaging $0.94 \mathrm{~kg} / \mathrm{min}$, i.e. a 0.61 dominance ratio. In the studied sample, the allele hd frequency was 0.39 and the marginal heritability 0.48 . We conclude that selecting goats with high milk flow is possible and useful. This selection may be organized within the sire dams sub-population (considering the best indexes in protein production and protein content), in order to detect dams with a milk flow higher than $1.46 \mathrm{~kg} / \mathrm{min}$ (supposed hd/ hd) and which rapidly produce young males having a large probability of being hd/hd.

RICORDEAU G., BOUILLON J., LEROY P., ELSEN J.M., 1990. Déterminisme génétique du débit de lait au cours de la traite des chèvres. INRA Prod. Anim. $3(2) 121-126$ 